\title{
Questioning the legitimacy of bariatric surgery: a qualitative analysis of individuals from the community who qualify for bariatric surgery
}

\author{
Grace F. Chao $0^{1,2,3}$ - Adrian Diaz ${ }^{1,2,4} \cdot$ Amir A. Ghaferi ${ }^{5,6} \cdot$ Justin B. Dimick $^{5,6} \cdot$ Mary E. Byrnes $^{5,6}$
}

Received: 30 August 2021 / Accepted: 6 December 2021 / Published online: 3 January 2022

This is a U.S. government work and not under copyright protection in the U.S.; foreign copyright protection may apply 2021

\begin{abstract}
Background Little is known about how individuals in the community who qualify for bariatric surgery perceive it and how this affects their likelihood to consider it for themselves. This study is the first qualitative study of a racially and ethnically diverse cohort to understand perceptions of bariatric surgery.

Methods We designed a descriptive study to understand attitudes about bariatric surgery. We interviewed 32 individuals who met NIH criteria for bariatric surgery but have never considered bariatric surgery. We purposively sampled to ensure the majority of participants were non-white. Using an Interpretive Description framework, an exploratory, iterative method was used to code interviews and arrive at final themes.

Results Participants self-identified as $88 \%$ female, 75\% Black, 3\% Hispanic, 3\% Pacific Islander, and 19\% white. Three major themes emerged from our data regarding legitimacy of bariatric surgery. First, participants perceived bariatric surgery to be something commercialized rather than needed treatment. They equated bariatric surgery with "botulism of the lips" or "cool sculpting." Second, an important contributor to the lack of legitimacy as a medical treatment was that many had not heard about bariatric surgery before from their doctors. Doctors were trusted sources for legitimate information about health. Lastly, conflicting information over bariatric surgery-related diet and weight loss further diminished the legitimacy of bariatric surgery. As one participant reflected about pre-operative weight loss requirements, "[If] I'm going to do that, I might as well just keep losing the weight. Why even go do the surgery?".

Conclusion Though bariatric surgery is a safe, effective, and durable therapy for patients with obesity, the majority of individuals we interviewed had concerns over the legitimacy of bariatric surgery as a medical treatment. Moving forward in reaching out to communities about bariatric surgery, healthcare providers and systems should consider the presentation of information to attenuate these concerns.
\end{abstract}

Keywords Bariatric surgery utilization · Patient perspectives

An earlier version was accepted for podium presentation at the SAGES Annual Meeting 2021 and presented September 2, 2021 in Las Vegas, NV.

Grace F. Chao

grace.f.chao@yale.edu

1 National Clinician Scholars Program at the Institute for Healthcare Policy and Innovation, University of Michigan, 2800 Plymouth Road, Building 14, Suite G100, Ann Arbor, MI 48109, USA

2 Veterans Affairs Ann Arbor, Ann Arbor, MI, USA

3 Department of Surgery, Yale School of Medicine, New Haven, CT, USA
4 Department of Surgery, The Ohio State University College of Medicine, Columbus, OH, USA

5 Center for Healthcare Outcomes and Policy, University of Michigan, Ann Arbor, MI, USA

6 Department of Surgery, University of Michigan, Ann Arbor, MI, USA 
The two ways by which an individual may be connected to bariatric surgery are through provider referral and selfreferral [1]. While the majority of providers believe bariatric surgery is a valuable tool to treat of severe obesity, about $40 \%$ have cited concerns about post-operative complications and ineffective weight loss as their main barriers to referral [2]. Despite its well-established clinical effectiveness and safety for weight loss and obesity-related comorbidities [3-9], providers continue to have concerns over outcomes after bariatric surgery. If providers, individuals who are a part of the healthcare system, continue to have clinical concerns regarding bariatric surgery, it may be that individuals outside of healthcare have negative perspectives of bariatric surgery that prevent them from considering this as a possible therapy as well.

Little is known about how individuals who qualify for bariatric surgery perceive it and how this affects their likelihood to consider it for themselves. Prior studies have focused on characteristics of individuals who are active patients at specific primary care or weight loss clinics [10, 11] or patients already being evaluated for bariatric surgery [12-16]. However, such individuals are already connected to healthcare for obesity treatment and may differ from community members. Additionally, only a few studies include a significant number of patients who come from racially minoritized backgrounds [10, 13]. Lastly, nearly all studies utilized quantitative methods or survey methodology, consequently, important reasons why individuals do not pursue bariatric surgery remain uncaptured.

In this study, we engage a diverse range of individuals to understand their perceptions of bariatric surgery with a majority non-white sample. We designed an interpretive description where individuals partake in semi-structured interviews to capture attitudes about bariatric surgery and experiences with weight loss.

\section{Materials and methods}

\section{Study design}

This study is part of a larger qualitative exploration to investigate barriers to bariatric surgery in a sample of mostly non-white individuals. We use interpretive description as it is a non-categorical, qualitative methodology useful in clinical settings [17]. In interpretive description, study design includes the aim of how knowledge from data analysis can be applied in everyday practice. The study was approved by the University of Michigan Institutional Review BoardIRBMED (HUM\#00170297).

\section{Interview participants}

We used a purposive sampling strategy to recruit a racially and ethnically diverse cohort. We first partnered with a health center predominantly serving Black individuals, Western Wayne Family Health Centers (WWFHC) in Inkster, a community that is $73.7 \%$ Black [18]. All recruitment materials were developed in conjunction with our community partner and placed at the health center, libraries, and neighborhood stores. We supplemented our sampling strategy with outreach via the Michigan Medicine research portal. During recruitment, demographics of the cohort and data collected through interviews were reviewed to ensure sampling led to a cohort with mostly non-white participants. Prior to beginning data collection, we determined that our sample size would be 30 participants given the principles of information power [19] which include study aim, sample specificity, use of established theory, quality of dialogue, and analysis strategy. Our approach allowed for greater or fewer study participants as data are collected to ensure we had achieved information power. We found that 30 individuals provided strong information power at the end of our study and included an additional two individuals who had expressed interest in participating prior to our closing recruitment.

The participants of this study were from Southeastern Michigan. Inclusion criteria were being age 18 or older, meeting NIH criteria for bariatric surgery (BMI $\geq 40 \mathrm{~kg} / \mathrm{m}^{2}$ or BMI $\geq 35 \mathrm{~kg} / \mathrm{m}^{2}$ with an obesity-related comorbid condition), and never having considered bariatric surgery. Recruitment materials shared this study was interested in adults with weight and comorbidity criteria and did not mention "bariatric surgery." Screening for prior bariatric surgery was conducted when participants contacted the research team. Participants were compensated for their time upon completion of their interview. Participants were asked to selfidentify race/ethnicity (i.e., "Black," "African American," "Pacific Islander"), age, and insurance status. Participants were consented for use of this demographic data in reporting our findings and were given the opportunity to restrict reporting or collection of this data. We also asked that each participant provide a pseudonym in order to humanize the data reporting which is used throughout this manuscript.

\section{Interview procedures}

Interviews were conducted by two team members (GFC and MEB) from February to August 2020. Prior to COVID19 social distancing measures, authors met participants face-to-face at our community partner site or University of Michigan. After March 13, 2020, interviews were conducted over secure videoconference or telephone. All participants 
consented prior to a 35-90 min interview. Interviews were audio-recorded and transcribed verbatim by an external HIPAA-approved transcriptionist with identifying information redacted. Transcripts were reviewed for accuracy.

The objective of interviews was to understand participants' experiences with health and perceptions of bariatric surgery. With our community partner, we created a semistructured interview guide (Appendix 1). We used an active interview style [20] to allow participants to expand on ideas important to them.

\section{Data analysis}

An exploratory, iterative method was used to code transcripts. Team members (GFC and MEB) independently open coded four interviews examining for patterns, concepts, and constructs. The team met to discuss where codes converged and diverged to create a coding scheme. The codebook was tested on five transcripts and revised to capture the participant experience. Coded portions were examined as a team to refine codebook definitions. The first author then coded all interviews using eclectic coding [21], leaving room for data that did not fit the codebook and for in vivo coding. All transcribed interviews were coded using MaxQDA20 (VERBI Software, Berlin, Germany, 2019), a computerassisted qualitative data analysis software.

In this analysis, the research team met to discuss patterns and domains [22] present in coded data using an Interpretive Description framework [17]. From these patterns, we identified from participants' shared experiences that questions of "legitimacy" surrounding bariatric surgery affected their likelihood to consider. In this study, we report the findings from codes "legitimacy" and "trusted sources."

\section{Results}

Participant demographics of the thirty-two participants are described in Table 1. Of our participants, $72 \%$ self-identified as Black or African American, 3\% as Hispanic, 3\% as Pacific Islander, $3 \%$ as multiracial, and $19 \%$ as white (Table 1 ). The majority of our sample (88\%) identified as female, and there was a diversity of ages represented. In order to maintain anonymity, we do not list the occupations of our participants. Additional exemplar quotes are included in Table 2 unless otherwise noted. Two participants had never heard about bariatric surgery. All other participants had either known someone who elected to have bariatric surgery or knew about it from news and popular culture. Our analysis found that an important barrier to considering bariatric surgery for many participants was that they saw bariatric surgery as separate from mainstream healthcare and thus
Table 1 Participant characteristics participants self-identified all presented demographic information in the table

\begin{tabular}{lc}
\hline Characteristics & Participants \\
& $N=32$ \\
\hline Race/Ethnicity, No. (\%) & \\
Black or African American & $23(72 \%)$ \\
Hispanic & $1(3 \%)$ \\
Pacific Islander & $1(3 \%)$ \\
Mixed Race (Ghanaian White) & $1(3 \%)$ \\
White & $6(19 \%)$ \\
Sex, No. (\%) & \\
Female & $28(88 \%)$ \\
Male & $4(12 \%)$ \\
Age, y, Mean (SD) & $47.8(12.8)$ \\
Age Range, No. (\%) & \\
<45 & $15(47 \%)$ \\
45-65 & $13(41 \%)$ \\
$>65$ & $4(13 \%)$ \\
Occupation, No. (\%) & \\
Not working & $3(9 \%)$ \\
Retired & $2(6 \%)$ \\
Student & $1(3 \%)$ \\
Working full-time & $21(66 \%)$ \\
Working part-time & $5(16 \%)$ \\
Insurance Type, No. (\%) & \\
Private insurance & $18(56 \%)$ \\
Medicaid or medicare & $10(31 \%)$ \\
No insurance & $0(0 \%)$ \\
Did not specify & $4(13 \%)$ \\
\hline
\end{tabular}

illegitimate. Figure 1 includes the themes and subthemes from our analysis.

\section{"Quack science" for-profit}

Participants described bariatric surgery as a commodity rather than a legitimate medical therapy. That is to say, participants perceived bariatric surgery to be something commercial that could be purchased rather than treatment for a medical condition. When asked about bariatric surgery, participants equated it with other procedures that were outside of mainstream medicine such as "cool sculpting" which Paulina (44, Female, African American) was skeptical of: "I feel like if it's that easy or if it's that simple, then wouldn't everybody be doing it?" Participants made other associations between bariatric surgery and liposuction, laser eye surgery, lip injections, and laser eradication of abdominal wall fat. Another participant, Michelle (34, Female, Black), suggested: 
Table 2 Reasons why participants questioned the legitimacy of bariatric surgery with exemplar quotations

Theme Exemplars

Bariatric surgery was seen as for-profit Basically, okay, this is how it works, and this is what it is. And, you know, there's the success stories and whatnot, a few success stories. It was more of a sales rally than anything scientific. And it was kind of going, okay, I understand. This is a business and all that, but can you people please give me something I can work with? (Terrell, 49, Male, African American, describing a bariatric surgery information session that he attended)

I don't know if I've seen as many ads as there used to be. I felt like it was really big for a minute that a lot of the hospitals were pushing them. You know, like even [hospital] had their bariatric center. [Hospital] had a bariatric center... There was a lot of ads for it, and it felt like there were drugs that people were trying to come out with for a minute. (LaKeishia, 40, Female, Black)

I've always viewed bariatric surgery as cosmetic surgery... So I think people tend to do it because they felt like that's their last trolley, can't lose any weight. They can't seem to keep their weight down, so they need to be extreme to look good, you know... Right, it does seem like it's an optional, like you really don't need that. You could've got that tummy tuck. (Charmane, 57, Female, Black)

Participants' physicians had not ever discussed bariatric surgery as a therapy

Confusing information surrounding bariatric surgery-related diet and weight loss
I don't think doctors suggest it. Like my doctors never talked about it... Well, if doctors would mention it as an alternative, an option. I mean, I have been battling high blood pressure for years, probably 25 years. I have been overweight that long. I now have a CPAP. Why hasn't my doctor ever mentioned it?... No one has ever, nobody ever talks about it. (Charo, 66, Female, Hispanic)

So I think that, quite frankly, in my case, it would need to come from my doctor. So, you know, I see my PCP at least annually, and often I see her more often. And my weight is a constant discussion point for us, and she's been very vocal about what she wants me to do to tackle my weight. At no time has she said we want to consider surgery. And so in the absence of that, there's nothing that would make me look for it. I mean, there's nothing that would prompt me to research it. (Adrienne, 57, Female, Black)

I'm not like knocking the surgery at all...Like and again, I keep going back to the show because that's the only thing I really know about it. And it's like he says, okay, you have to lose this much in a month. And I'm like so if you could do that in a month, why not just keep that up? You know, like is that healthy?...Like just if you can do that for one month, do it for like four more, you know? (Michelle, 34, Female, Black)

I felt it was probably a shortcut to weight loss as opposed, I mean, you do have to change your eating habits, because depending on the type of bariatric surgery, there's certain foods you cannot eat. Now, she, say the one where they reduce the size of your stomach, so you're almost forced to kind of change eating habits. But then again, some people go back to old eating habits, and that's how you're gaining the weight back. So that's the part that's almost like contradictory to me. (Sandra, 64, Female, African American)
Fig. 1 Themes and subthemes for how participants viewed bariatric surgery as illegitimate. The top row includes the themes, and the second and third rows include subthemes

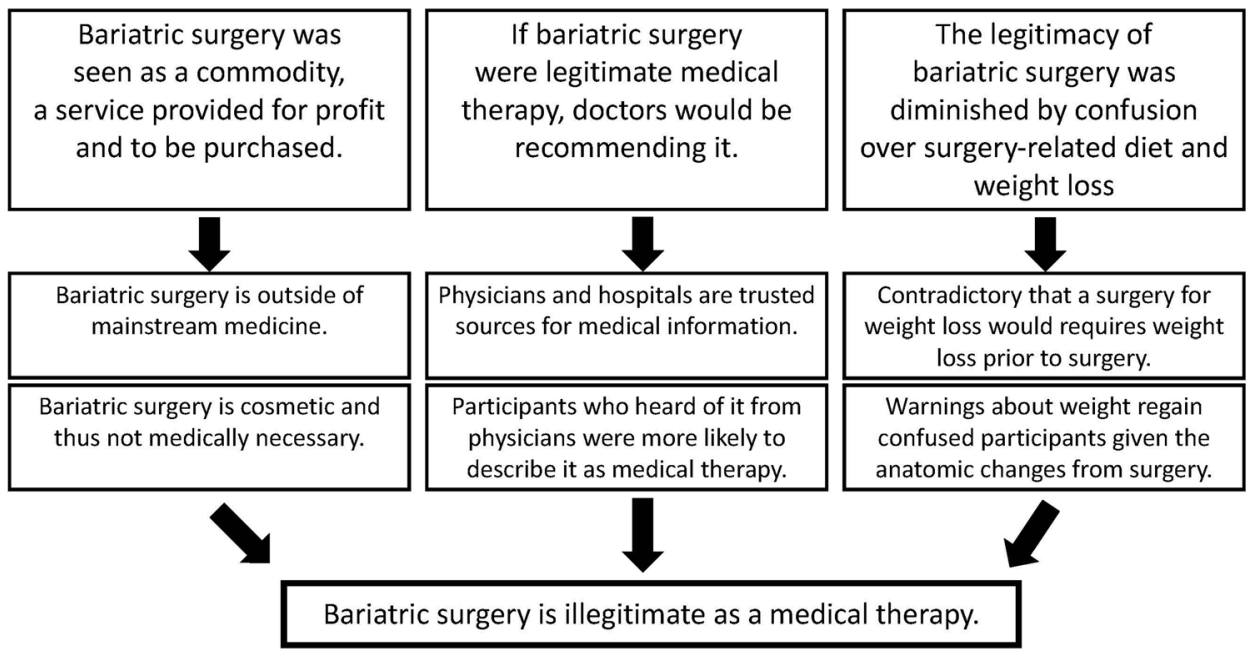

saying, 'oh, you go to Mexico, something like that.' Like if it's safe and it gets people off medications or better numbers. 
As Michelle describes, bariatric surgery was so far outside of mainstream medicine to some people that you might even have to leave the country to get it done. Thus, participants viewed bariatric surgery as a commodity being sold to them for-profit rather than medical treatment.

Participants also noted how bariatric surgery was done at "centers" which further separated bariatric surgery from mainstream medicine. One participant (Adrienne, 57 , Female, Black) who called bariatric surgery a "quack science" also shared:

It seems, maybe I put it over there with I don't know, botulism in the lips...those treatment centers that I don't trust. I have similar views when it comes to laser surgery to correct vision problems. And I think, oh, they're going through these weird things that may not be real hospitals, and it seems profit-driven...There's centers, which doesn't give me that warm confidence that it's my doctor's practice.

When discussing more positive feelings about seeking out bariatric surgery, Terrell (49, Male, African American) mentioned he would seek doctors: who are connected to hospitals. That was important to me, because, you know, you see stuff on the news and like, oh, he's an independent doctor in his clinic, and something went wrong, very wrong. Calling bariatric surgery clinics "centers" further separated bariatric surgery from legitimate medicine.

A few participants also equated bariatric surgery to cosmetic procedures which could be done by medical professionals or not. As Sandra shared (64, Female, African American):

I know at one time, when bariatric surgery first came out, it was a controversy on where your insurance would pay for it. Because I guess, because they said if it was for cosmetic purposes, I guess, your insurance wasn't going to pay for it, so I don't know how it is now.

These participants felt bariatric surgery was for cosmetic reasons, further making it seem like something elective or a luxury one could purchase rather than needed medical treatment.

\section{Why hasn't my doctor ever mentioned it?}

An important contributor to the lack of legitimacy of bariatric surgery as a medical treatment was that many had not heard about it before from their doctors. Almost all participants who had a primary care physician had discussed their weight in some capacity with them. Vicky (49, Female, Multiracial) was skeptical of bariatric surgery as a legitimate treatment:
But I'm also treated for obesity, but [my doctors] haven't referred me anywhere for obesity. They never, I don't know how trusting they are of those things because they never referred me anywhere else for obesity, none of them have. And, but they've mentioned obesity, I've been treated for obesity, but they've never treated me like given me a referral to a bariatric surgeon.

Conversely, participants whose primary care physicians had mentioned bariatric surgery before spoke about bariatric surgery's potential benefits as a medical treatment, "to promote better health for myself' (Carolyn, 66, Female, African American).

\section{Physicians and hospitals as trusted sources}

Participants were more likely to consider bariatric surgery as an option when doctors or well-known hospitals presented it. This was due to their trust in these individuals and organizations to care for their health and to give unbiased information. As Adrienne (57 years old, Female, Black), the participant who had equated bariatric surgery with botulism of the lips, shared:

If my doctor, if she were to come to me and say, 'This is bariatric surgery. I'd like you to consider it. These are the potential dangers.' Just as they gave me the potential dangers of, you know, other treatment plans.

"This is why I think it's a good idea for you, this is how we'll do it," and I would be likely to consider it... if my doctor were to give me something I would trust it, and she would say read it, and I would, and I would go forward. But on my own, I wouldn't look for something.

Similarly, when asked where she would go for trusted information about her body or health, Amber (33, Female, African American) immediately said "My doctor probably or my gynecologist, or my psychiatrist." Other participants named specific hospitals which were "the top type places" they trusted as whose websites they would use as a starting point for information (Julie, 42, Female, Black).

Participants whose physicians had talked to them about bariatric surgery described it as a "medical therapy." For example, Latoria (33, Female, Black) whose doctor had brought up bariatric surgery to her twice said she would consider bariatric surgery if.

my Alc is still ten, nine, ten, and, you know, great, it came down significantly, but it hasn't moved in the last 2 years. Then I know that, yeah, I feel at that particular moment, then we can talk about [bariatric surgery]. 
Other participants like Latoria named clinical outcomes that could improve with bariatric surgery when their doctor had mentioned it to them before.

In contrast, the media was sometimes seen as untrustworthy. As Julie (42, Female, Black) went on to explain, she felt getting information from a source like Mayo Clinic was different than from watching the television show 1000lb Sisters:

Of course, she's lost like 100 pounds or something within, you know, a very short amount of time. So you do see that. Like you see that very glossy Hollywood kind of overview, and I think that the responsible thing to do is to present both sides.

When experiences with bariatric surgery were portrayed on television, participants felt that likely what being shown was only the positive portions which made them skeptical of bariatric surgery overall. Other exemplar quotes for this theme regarding trusted sources are found in Table 3.

\section{Confusion over bariatric surgery-related diet and weight loss}

Lastly, for a few participants, the legitimacy of the procedure was diminished by confusion over bariatric surgery-related diet and weight loss. Many participants noted that it seemed weight loss was required prior to surgery. This was confusing to participants because if surgery was meant to help with weight loss, why did patients have to lose weight before surgery as a requirement? As Paulina (44, Female, African American) reflected on the experiences of individual on the television show My 600-lb Life:

They want you to lose a certain amount of weight before you can get it going and all of that. And I feel like if I'm going to do that, I might as well just keep losing the weight. Why even go do the surgery?
Another source of confusion was the warnings of weight regain especially because it seemed impossible to reverse the effects of the surgery with so little stomach available to fill with food. When considering life after bariatric surgery, some participants felt the information that they knew about bariatric surgery contradicted itself.

\section{Discussion}

To our knowledge, this is the first study to understand how individuals from the community who qualify but not have pursued bariatric surgery perceive bariatric surgery with regard to legitimacy as a medical therapy. Participants shared they questioned the legitimacy of bariatric surgery due to its seemingly for-profit nature, because their physicians had not discussed it with them, and because of ideas about diet and weight loss related to bariatric surgery which seemed to contradict each other. From these perceptions, we also identified that when participants heard about bariatric surgery from these trusted sources-physicians and hospitals-they were more likely to consider bariatric surgery.

The effect of physicians talking about bariatric surgery to encourage individuals towards considering surgery is aligned with prior research. In a study of patients with BMI $>35 \mathrm{~kg} / \mathrm{m}^{2}$, individuals were 5 times more likely to consider bariatric surgery when recommended by their primary care physician [10]. Despite the importance of trusted information from providers and hospitals, a study of 14,143 patients with obesity found weight management counseling declined from 33 to $21 \%$ from 2008 to 2013 [23]. Prior studies have identified that completion of bariatric CME [24] and obesity-related training [25] increases the likelihood of referral. Additionally, barriers to provider referral include unfamiliarity with NIH guidelines [24], discomfort counseling on obesity management [26], and discomfort explaining bariatric procedures [27].

Table 3 Trusted sources of information exemplar quotations

\begin{tabular}{ll}
\hline Theme & Exemplars \\
$\begin{array}{l}\text { Physicians and hospitals were trusted sources of information that legiti- } \\
\text { mized bariatric surgery as a therapy }\end{array}$ & $\begin{array}{c}\text { I'm not totally against it for me because I'm, I want to improve my } \\
\text { health conditions. (Kim, 55, Female, African American) } \\
\text { And so my family doctor, you know, did bring up the fact of, there are } \\
\text { different procedures on the market right now. So before, initially, I } \\
\text { just always thought it was, you know, maybe for someone that was } \\
\text { like severely obese, and I don't consider myself to be that way... So } \\
\text { I guess my thoughts of it are, I'm kind of like on the fence because... } \\
\text { if it's kind of like a life-death-maybe situation, then I think people } \\
\text { should consider doing it. (Jen, 41, Female, Black) } \\
\text { [My doctor] just basically said it was like an option to help me like } \\
\text { lose some weight if I chose to go that route...And he was like, and } \\
\text { he weighed me, and he looked at my condition. And he was like } \\
\text { maybe talking about I was prediabetic and said, you know, I should } \\
\text { try losing some weight. (Myron, 46, Male, Black) }\end{array}$
\end{tabular}


A targeted intervention for physicians focused on these components (risks, benefits, perioperative experience for patients, and post-operative course) as well as how to discuss bariatric surgery with patients as part of routine care could increase physician recommendation of bariatric surgery. As our study has shown, this is key to legitimizing bariatric surgery for individuals in the community. Increasing physician discussions with patients regarding bariatric surgery may have significant widespread impact as one physician can influence an entire group of patients. The authors believe that as obesity affects all organ systems, physicians from all fields (cardiology, endocrinology, family medicine, obstetrics and gynecology, orthopedic surgery, primary care, pulmonology, etc.) are all crucial to helping our patients better understand the role that bariatric surgery can play in their health. Thus, the effectiveness of such an educational intervention as well as the scale of impact could start to address the current less than $1 \%$ utilization of bariatric surgery by eligible individuals [28].

Other aspects of beliefs about bariatric surgery have been previously found to affect a patient's likelihood of considering bariatric surgery. A narrative review [12] found that fear of surgery [29] and postoperative complications [14, 29, 30], perception of surgery as "extreme" [14], and concerns regarding postoperative restrictions [30], weight regain [14], and lack of control over weight loss [14] were barriers to considering bariatric surgery. Our study highlights the importance of questions of legitimacy of bariatric surgery when considering individuals who have not yet engaged with healthcare regarding bariatric surgery, a barrier not previously described among individuals who are already established patients.

Additionally, physicians must consider the social context in which individuals live to best engage individuals in thinking about bariatric surgery. In our society which is characterized by consumerism and obesity stigma, we are inundated with advertisements which include a large number regarding promised weight loss remedies. In 2019 alone, the United States weight loss industry was estimated to be worth $\$ 78$ billion [31]. The weight loss industrial complex includes everything from meal services to pharmaceutical remedies to options identified by participants such as cool sculpting. Separating bariatric surgery from commercialized notions of weight loss will require work on our part. Possible future directions may include renaming "centers" as "clinics" or other methods of making it more clear that physicians are offering a medical treatment.

Our study has limitations. First, our recruitment strategy was designed for outreach to neighborhood residents through postings in libraries and stores, but many of our participants were recruited through our community partner, WWFHC. Thus, many participants were already connected to healthcare in some way. There may be different beliefs held by individuals who do not engage healthcare at all. Despite this, our sample has diversity by age, race, insurance status, and occupation.
Second, almost all participants had heard of bariatric surgery. It is possible that individuals who have never heard about bariatric surgery may have different facilitators and barriers to considering bariatric surgery. However, our recruitment materials do not use the words "bariatric surgery," so it is unlikely that our participants chose to participate based on strong opinions about bariatric surgery which would bias our results. Finally, the sample was recruited from Southeastern Michigan, so our findings may not be generalizable to other contexts.

\section{Conclusion}

This qualitative study of individuals who would qualify for bariatric surgery found that a major barrier to considering bariatric surgery was concerns over its legitimacy as a medical treatment. Moving forward in reaching out to communities to share information about bariatric surgery, healthcare providers and systems should consider the presentation of information as well as the content in order to attenuate these concerns.

\section{Appendix 1. Semi-structured interview guide}

1. Healthcare

a. Perceptions of healthcare

i. Some doctors think bariatric surgery is a way to treat obesity and associated medical issues. What do you think about that?

ii. Have you ever considered bariatric surgery? What are some things that make it difficult to get bariatric surgery?

iii. When would you consider surgery?

iv. Is there any reason why you wouldn't get surgery?

v. Tell me about your experience in the healthcare system.

vi. Was there ever a time when you needed healthcare but didn't go? Why?

vii. What does healthcare mean to you?

viii. What do you think about doctors? What about nurses?

1. Have you ever been mistreated or heard of anyone being mistreated? Walk me through what happened.

b. Self-efficacy

i. How would you rate your health today? 
2. Self

a. Body/trustworthy knowledge

i. Can you tell me the things that you love about yourself?

ii. Where do you get information about your body/health that you trust?

iii. Can you tell me about a time a doctor said something about your body? What do you think about what they said?

b. Intersectionality

i. Can you tell me about a time that you were treated with less respect based on your identity? Which aspects?

ii. Threatened? Treated as less smart? Received worse service?

3. Society

a. Media

i. Overweight and obesity is an issue often mentioned in the media. Has weight ever been something that you, family, or friends were worried about or talked about?

ii. Tell me about any advertisements or any articles you have seen about weight. Have you seen advertisements by doctors or clinics about weight? How did they make you feel? If you discussed them with anyone, what was that conversation like?

iii. How do concerns about weight affect the way you eat or do physical activity?

4. Healthcare revisited

a. How might your health be affected by your weight? Does weight relate to blood pressure? diabetes? sleep? breathing? joint problems?

b. What kinds of help / support would you need in order to get bariatric surgery both before and after?

c. What might interfere with your change in lifestyle after bariatric surgery?

d. Do you worry about paying for healthcare?

Acknowledgements We would like to thank Western Wayne Family Health Centers-Inkster for partnering with us to amplify Black voices in research. WWFHC was vital in helping us to develop our interview instrument and recruitment materials and to reach out to the community.

\section{Declarations}

Disclosures Dr. Chao receives funding from the Veterans Affairs Center for Clinical Management Research, VA Ann Arbor Healthcare System; this work does not represent the views of the United States government nor the Department of Veterans Affairs. Dr. Diaz receives funding from the Veterans Affairs Center for Clinical Management Research, VA Ann Arbor Healthcare System; this work does not represent the views of the United States government nor the Department of Veterans Affairs. Dr. Ghaferi is supported through grants from the Agency for Healthcare Research and Quality (Grant \#: 5K08HS02362 and P30HS024403) and a Patient Centered Outcomes Research Institute Award (CE-1304-6596). Dr. Ghaferi receives salary support from Blue Cross Blue Shield of Michigan as the Director of the Michigan Bariatric Surgery Collaborative. Dr. Dimick receives grant funding from the NIH, AHRQ, and BlueCross BlueShield of Michigan Foundation. Dr. Dimick is a cofounder of ArborMetrix, Inc, a company that makes software for profiling hospital quality and efficiency. Dr. Byrnes has no conflicts of interest to report.

\section{References}

1. Jabour SM, Griauzde DH, Chao GF, Ehlers AP (2021) How do patients access bariatric surgery? an analysis of referrals to a large academic medical center. Obes Surg. https://doi.org/10.1007/ s11695-021-05584-7

2. Conaty EA, Denham W, Haggerty SP, Linn JG, Joehl RJ, Ujiki MB (2020) Primary care physicians' perceptions of bariatric surgery and major barriers to referral. Obes Surg 30:521-526

3. Arterburn D, Wellman R, Emiliano A, Smith SR, Odegaard AO, Murali S, Williams N, Coleman KJ, Courcoulas A, Coley RY, Anau J, Pardee R, Toh S, Janning C, Cook A, Sturtevant J, Horgan C, McTigue KM, Collaborative PCBS (2018) Comparative effectiveness and safety of bariatric procedures for weight loss: A PCORnet cohort study. Ann Intern Med 169:741-750

4. McTigue KM, Wellman R, Nauman E, Anau J, Coley RY, Odor A, Tice J, Coleman KJ, Courcoulas A, Pardee RE, Toh S, Janning CD, Williams N, Cook A, Sturtevant JL, Horgan C, Arterburn D, PCBS Collaborative (2020) Comparing the 5-year diabetes outcomes of sleeve gastrectomy and gastric bypass: the national patient-centered clinical research network (PCORNet) bariatric study. JAMA Surg. https://doi.org/10.1001/jamasurg.2020.0087

5. Salminen P, Helmio M, Ovaska J, Juuti A, Leivonen M, PeromaaHaavisto P, Hurme S, Soinio M, Nuutila P, Victorzon M (2018) Effect of laparoscopic sleeve gastrectomy vs laparoscopic Rouxen-Y gastric bypass on weight loss at 5 years among patients with morbid obesity: the SLEEVEPASS randomized clinical trial. JAMA 319:241-254

6. Peterli R, Wolnerhanssen BK, Peters T, Vetter D, Kroll D, Borbely Y, Schultes B, Beglinger C, Drewe J, Schiesser M, Nett P, Bueter M (2018) Effect of laparoscopic sleeve gastrectomy vs laparoscopic Roux-en-Y gastric bypass on weight loss in patients with morbid obesity: the SM-BOSS randomized clinical trial. JAMA 319:255-265

7. Arterburn DE, Johnson E, Coleman KJ, Herrinton LJ, Courcoulas AP, Fisher D, Li RA, Theis MK, Liu L, Fraser JR, Haneuse S (2020) Weight outcomes of sleeve gastrectomy and gastric bypass compared to nonsurgical treatment. Ann Surg. https://doi.org/10. 1097/SLA.0000000000003826

8. Chhabra KR, Telem DA, Chao GF, Arterburn DE, Yang J, Thumma JR, Ryan AM, Blumenthal B, Dimick JB (2020) Comparative safety of sleeve gastrectomy and gastric bypass: an 
instrumental variables approach. Ann Surg. https://doi.org/10. 1097/SLA.0000000000004297

9. Chao GF, Chhabra KR, Yang J, Thumma JR, Arterburn DE, Ryan AM, Telem DA, Dimick JB (2020) Bariatric surgery in medicare patients: examining safety and healthcare utilization in the disabled and elderly. Ann Surg. https://doi.org/10.1097/SLA.00000 00000004526

10. Wee CC, Huskey KW, Bolcic-Jankovic D, Colten ME, Davis RB, Hamel M (2014) Sex, race, and consideration of bariatric surgery among primary care patients with moderate to severe obesity. J Gen Intern Med 29:68-75

11. Arterburn D, Westbrook EO, Terrell A (2013) Weight control practices of severely obese patients who are not seeking bariatric surgery. Obesity (Silver Spring) 21:1509-1513

12. Imbus JR, Voils CI, Funk LM (2018) Bariatric surgery barriers: a review using Andersen's model of health services use. Surg Obes Relat Dis 14:404-412

13. Stanford FC, Jones DB, Schneider BE, Blackburn GL, Apovian CM, Hess DT, Chiodi S, Robert S, Bourland AC, Wee CC (2015) Patient race and the likelihood of undergoing bariatric surgery among patients seeking surgery. Surg Endosc 29:2794-2799

14. Funk LM, Jolles S, Fischer LE, Voils CI (2015) Patient and referring practitioner characteristics associated with the likelihood of undergoing bariatric surgery: a systematic review. JAMA Surg 150:999-1005

15. Wee CC, Hamel MB, Apovian CM, Blackburn GL, BolcicJankovic D, Colten ME, Hess DT, Huskey KW, Marcantonio ER, Schneider BE, Jones DB (2013) Expectations for weight loss and willingness to accept risk among patients seeking weight loss surgery. JAMA Surg 148:264-271

16. Bergmann KL, Cox SJ, Tabone LE (2017) Influence of a rural environment on patient access and outcomes for bariatric surgery. Surg Obes Relat Dis 13:632-636

17. Thorne SE (2016) Interpretive description: qualitative research for applied practice, 2 nd edn

18. United States Census Bureau (2019) QuickFacts Inkster city, Michigan. https://www.census.gov/quickfacts/fact/table/inkstercit ymichigan/PST040219. Accessed 12 Aug 2021

19. Malterud K, Siersma VD, Guassora AD (2016) Sample size in qualitative interview studies: guided by information power. Qual Health Res 26:1753-1760
20. Holstein JA, Gubrium JF (1995) The active interview. SAGE Publications, Thousand Oaks, Calif

21. Saldaña $\mathbf{J}$ (2016) The coding manual for qualitative researchers, 3rd edn

22. Miles MB, Huberman AM, Saldaña J (2019) Qualitative data analysis : a methods sourcebook, 3rd edn. Sage Publication, Arizona

23. Fitzpatrick SL, Stevens VJ (2017) Adult obesity management in primary care, 2008-2013. Prev Med 99:128-133

24. Balduf LM, Farrell TM (2008) Attitudes, beliefs, and referral patterns of PCPs to bariatric surgeons. J Surg Res 144:49-58

25. Stanford FC, Johnson ED, Claridy MD, Earle RL, Kaplan LM (2015) The role of obesity training in medical school and residency on bariatric surgery knowledge in primary care physicians. Int J Family Med 2015:841249

26. Tork S, Meister KM, Uebele AL, Hussain LR, Kelley SR, Kerlakian GM, Tymitz KM (2015) Factors influencing primary care physicians' referral for bariatric surgery. JSLS. https://doi.org/10. 4293/JSLS.2015.00046

27. Perlman SE, Reinhold RB, Nadzam GS (2007) How do family practitioners perceive surgery for the morbidly obese? Surg Obes Relat Dis 3:428-433

28. Campos GM, Khoraki J, Browning MG, Pessoa BM, Mazzini GS, Wolfe L (2020) Changes in utilization of bariatric surgery in the United States from 1993 to 2016. Ann Surg 271:201-209

29. Fischer L, Wekerle AL, Sander J, Nickel F, Billeter AT, Zech U, Bruckner T, Muller-Stich BP (2017) Is there a reason why obese patients choose either conservative treatment or surgery? Obes Surg 27:1684-1690

30. Wharton S, Serodio KJ, Kuk JL, Sivapalan N, Craik A, Aarts MA (2016) Interest, views and perceived barriers to bariatric surgery in patients with morbid obesity. Clin Obes 6:154-160

31. Marketdata LLC (2021) The U.S. weight loss \& diet control market. Research and Markets

Publisher's Note Springer Nature remains neutral with regard to jurisdictional claims in published maps and institutional affiliations. 